\title{
The effect of Individualized Pre- and Post- Embryo Transfer acupuncture on implantation and clinical pregnancy rate
}

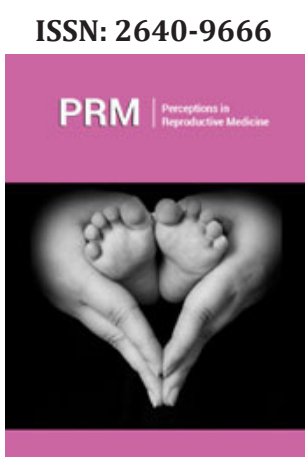

*Corresponding author: Ahmad Arabi, Department of Obstetrics and Gynecology, Wayne State University School of Medicine, Detroit, MI, USA

Submission: 海 January 28, 2021

Published: 酒February 17, 2021

Volume 4 - Issue 3

How to cite this article: Ahmad Arabi, Katherine EB, Grimm L, Mowl S, Beltsos A, et al. The effect of Individualized Pre- and Post-Embryo Transfer acupuncture on implantation and clinical pregnancy rate Perceptions in Reproductive Medicine. 4(3). PRM. 000589. 2021.

DOI: 10.31031/PRM.2021.04.000589

Copyright@ Ahmad Arabi, This article is distributed under the terms of the Creative Commons Attribution 4.0 International License, which permits unrestricted use and redistribution provided that the original author and source are credited.
Ahmad Arabi MD ${ }^{1 *}$, Brown $\mathrm{K} \mathrm{BS}^{2}$, Grimm L MA², Mowl S BS ${ }^{3}$, Beltsos A MD ${ }^{4}$, Kamel L DAOM ${ }^{5}$ and Jeelani R MD ${ }^{4}$

${ }^{1}$ Department of Obstetrics and Gynecology, Wayne State University School of Medicine, Detroit, MI, USA

${ }^{2}$ Wayne State University School of Medicine, Detroit, MI, USA

${ }^{3}$ Department of Psychology, University of Michigan, Department of Psychology, Flint, MI, USA

${ }^{4}$ Vios Fertility Institute, Chicago, IL, USA

${ }^{5}$ Allied Modern Health, Chicago, IL, USA

\begin{abstract}
Objective: The purpose of this study is to examine the implantation and clinical pregnancy rates of patients undergoing elective, individualized pre-and post-embryo transfer acupuncture compared to
\end{abstract} receiving no acupuncture on day of transfer.

Design: In this retrospective chart review, 430 records from May 2018-September 2020 were reviewed to assess the effectiveness of the elective, individualized pre- and post-embryo transfer acupuncture $(\mathrm{n}=262)$ on implantation rate (positive $\beta$-hCG test) and clinical pregnancy rate (intrauterine gestational sac confirmed on ultrasound) compared to those patients who did not receive acupuncture $(n=168)$.

Methods: Patients meeting Vios Fertility Institute's clinical standards for having a frozen embryo transfer can elect to have individualized pre- and post-embryo transfer acupuncture performed by licensed acupuncturists on-site, 30 minutes before and immediately after embryo transfer. All single embryo transfers with known Pre-Implantation Genetic Testing (PGT), acupuncture, and Endometrial Receptivity Array (ERA) data, as well as pregnancy outcomes, were queried for this study. Transfers were conducted at a single fertility center in Chicago, IL. A binomial logistic regression was performed to determine the effects of acupuncture on the rate of implantation and clinical pregnancy. The model was used to estimate the OR of implantation and clinical pregnancy rates after adjusting for age, Body Mass Index (BMI), AntiMullerian Hormone (AMH), PGT, ERA, day of transfer, and embryo morphology.

Result: The percentage of embryo transfers resulting in implantation was positively associated with the use of acupuncture. $76.7 \%$ in the acupuncture group compared to $65.2 \%$ in the control group in the PGT tested subset of embryos, and $62.1 \%$ compared to $51.52 \%$ in the embryos without PGT. This difference was found to continue throughout clinical pregnancy rates $(71.1 \%$ compared to $56.5 \%$ in the subset of PGT tested embryos; $55.3 \%$ compared to $46.5 \%$ in the subset without PGT). A multivariate logistic regression model was then used to isolate the impact of acupuncture from other confounding variables. When compared with the group without acupuncture, significantly increased implantation rates were observed in the acupuncture group (OR 1.64, 95\% CI 1.04-2.58). Clinical pregnancy rates were also noted to be significantly increased (OR 1.64, 95\% CI 1.05-2.56).

Conclusion: Overall, individualized acupuncture performed on the day of embryo transfer was significantly associated with higher implantation and clinical pregnancy rates. Follow-up research will investigate the difference in live birth rates and cost-effectiveness of additional treatments

\section{Introduction}

Acupuncture is a practice that originated in China approximately 3,000 years ago. Acupuncture was first described within the framework of diagnosis and treatment in The Yellow Emperor's Classic of Internal Medicine, dating back to 100 BCE [1]. Within Traditional Chinese Medicine (TCM), needles are inserted at specific points (acupoints) along a meridian (energy channel), through which qi (life force energy) flows. Within TCM, it is believed that needling specific points affect specific organ systems, primarily by removing blocks, or stagnation, on a meridian. The overall goal of acupuncture is to bring the body into balance, such that disease and disorders do not occur [2]. Acupuncture was brought to America and Britain's attention when Ten Rhijne, a European physician, created the first medical description of acupuncture in 1680 [1]. Following this introduction to the West, acupuncture publications began appearing in modern medicine journals during the first half of the $19^{\text {th }}$ 
century. Until recent years, western medicine had the consensus that acupuncture was a sham. There is now significant evidence showing both its efficacy and effectiveness. Acupuncture has a wide scope of conditions that have been proven to be successfully treatable. Conditions range from emotional, psychological, and musculoskeletal conditions, to immunity, nausea, migraines, and infertility [3]. The use of acupuncture in modern medicine has become increasingly common, as it allows for a whole-body approach, filling the gaps that modern medicine is not equipped to fill. During embryo transfer procedures, there is minimal control physicians have over implantation success. It has become common for acupuncture to be offered to patients seeking in-vitro fertilization (IVF), with the purpose of increasing implantation rates [4]. Multiple physiological mechanisms have been considered to be influenced by acupuncture treatment.

A randomized control trial examined the effects of acupuncture treatment before and after an embryo transfer procedure on women undergoing IVF [5]. The women in the experimental group were shown to have significantly increased implantation success rates and significantly lower anxiety levels [5]. Another randomized control trial examined the effects of acupuncture on implantation success and found that acupuncture treatment before and after the embryo transfer procedure resulted in a significant increase in success rates [6]. A systematic review and meta-analysis concluded that acupuncture may be effective when compared to a nontreatment control, but when compared to a sham treatment control, the effect is not as strong [7]. There are confounding variables involved in the use of acupuncture treatments in clinical studies. There is a need for larger studies and improved methodologies to conclude a more accurate consensus on the use of acupuncture for implantation success rates while controlling for confounders.

One mechanism by which embryo transfer implantation rates are believed to be improved by acupuncture is via modulating $\beta$-endorphin secretion [8,9]. These then increase pulsatile release of gonadotropin releasing hormone $(\mathrm{GnRH})$, which increases pituitary secretion of gonadotropin and thereby steroid release [1012]. In addition, acupuncture may downregulate sympathetic tone, allowing for increased blood flow to the uterus and ovaries [13]. These conditions may increase endometrial growth and thickness, creating a more hospitable environment for implantation. While these proposed mechanisms may be responsible for the positive effect of acupuncture on implantation rates, alternative explanations include psychological factors, like stress reduction, or placebo effects [4]. The purpose of this study is to examine implantation success rates of patients undergoing elective, individualized acupuncture pre- and post-embryo transfer versus no acupuncture on the day of transfer.

\section{Material and Methods}

\section{Inclusion and exclusion criteria}

In order to better assess the effect of acupuncture alone on implantation rates, an attempt was made to correct for confounding variables that could potentially impact outcomes. Only single frozen embryo transfers were considered for this study, as multiple embryo transfers may impact outcomes. Only frozen embryo transfers were considered due to the potential difference between fresh and frozen implantation rates [14]. All single embryo transfers with known PGT, acupuncture, endometrial receptivity array (ERA) data, as well as pregnancy outcomes were queried for this study. Transfers were conducted at a single fertility center in Chicago, IL, Vios Fertility Institute.

\section{Cycle protocols}

Standard stimulation protocols were used for ovarian hyperstimulation using recombinant or purified Follicle Stimulating Hormone (FSH) or Human Menopausal Gonadotropin (HMG). A GnRH agonist for long downregulation or an antagonist protocol was used to prevent premature Luteinizing Hormone (LH) surge and ovulation. Because there is no significant difference in outcomes between different protocols, [15] patient to patient differences in these protocols were not used as reasons for exclusion. In this fertility center, intracavitary conditions such as submucosal fibroids, endometrial polyps, and intrauterine adhesions are evaluated and corrected prior to embryo transfer.

\section{Acupuncture}

All patients meeting Vios' clinical standards for having a frozen embryo transfer can elect to have individualized pre- and postembryo transfer acupuncture performed on-site 30 minutes before and immediately after embryo transfer. Licensed acupuncturists perform these 2 treatments on-site at Vios Fertility Institute. The patient's past medical and cycle history are reviewed to inform the direction of their individualized acupuncture treatments. No protocols were used to ensure that patients received treatment appropriate for their case.

\section{Age groups}

A total of 430 embryo transfers, from 2018-2020, met the criteria and were included in the study. They were divided into five age groups $-<35,35$ to 37,38 to $40,41-42$, and $>42$ years old as determined by the Society of Assisted Reproductive Technology [16].

\section{Implantation, clinical pregnancy rates}

Implantation is defined as a positive serum $\beta$-hCG level within 14 days of embryo transfer, and clinical pregnancy was identified as a visualized intrauterine gestational sac or fetal pole on ultrasound, regardless of whether they progressed any further.

\section{Potential confounders}

Multiple factors identified as potential confounders with an effect on embryo implantation were accounted for in this study. These variables include age, BMI, AMH, PGT, ERA, embryo morphology, and day of transfer [17]. These variables were included into a multivariate logistic regression model to adjust for the potential confounding effect. 


\section{Statistical analysis}

A binomial logistic regression was performed to determine the effects of acupuncture on the rate of implantation and clinical pregnancy. The model was used to estimate the OR of implantation and clinical pregnancy rates after adjusting for age, BMI, AMH, PGT, ERA, day of transfer, and embryo morphology. The logistic regression model was statistically significant, $\chi^{2}(4)=56.37, \mathrm{p}<.001$. The model explained $17.0 \%$ (Nagelkerke R2) of the variance in implantation rates and correctly classified $70.2 \%$ of cases. Sensitivity of the model was $91.1 \%$, specificity was $30.4 \%$. All statistical analyses were performed by using SPSS Survey Procedures. Statistical significance was defined by a two-sided test with a $\mathrm{p}$ value of $<0.05$.

\section{Result}

\section{Group distribution and demographics}

Table 1: Demographics and baseline characteristics. PGT, ERA, Embryo grade, and day of transfer are shown as $\mathrm{n}$ (\% of total); all others shown as mean (SD).

\begin{tabular}{|c|c|c|c|c|}
\hline & & Total $(n=430)$ & Acupuncture $(n=262)$ & No Acupuncture $(n=168)$ \\
\hline \multicolumn{2}{|c|}{ Age (years) } & $35.8(4.7)$ & $35.2(4.70)$ & $36.6(4.57)$ \\
\hline \multicolumn{2}{|c|}{ BMI $\left(\mathrm{kg} / \mathrm{m}^{2}\right)$} & $27.1(3.32)$ & $27.2(3.98)$ & $26.89(1.90)$ \\
\hline \multicolumn{2}{|c|}{ AMH (ng/mL) } & $27.1(3.32)$ & $27.2(3.98)$ & $26.89(1.90)$ \\
\hline \multicolumn{5}{|l|}{ PGT } \\
\hline & No & $202(47.0 \%)$ & $103(39.3 \%)$ & $99(58.9 \%)$ \\
\hline & Yes & $228(53.0 \%)$ & $159(60.7 \%)$ & $69(41.1 \%)$ \\
\hline \multicolumn{5}{|l|}{ ERA } \\
\hline & No & $344(80.0 \%)$ & $206(78.6 \%)$ & $138(82.1 \%)$ \\
\hline & Yes & $86(20.0 \%)$ & $56(21.4 \%)$ & $30(17.9 \%)$ \\
\hline \multicolumn{5}{|c|}{ Embryo Grade } \\
\hline & Unknown & $170(39.5 \%)$ & $103(39.3 \%)$ & 67 (39.9\%) \\
\hline & Poor & 0 & 0 & 0 \\
\hline & Fair & $97(22.6 \%)$ & $64(24.4 \%)$ & $33(19.6 \%)$ \\
\hline & Good & $163(37.9 \%)$ & $95(36.3 \%)$ & $68(40.5 \%)$ \\
\hline \multicolumn{5}{|c|}{ Day of Transfer } \\
\hline & 3 & $9(2.1 \%)$ & $6(2.3 \%)$ & $3(1.8 \%)$ \\
\hline & 5 & $343(79.8 \%)$ & $214(81.7 \%)$ & $129(76.8 \%)$ \\
\hline & 6 & $51(11.9 \%)$ & $37(14.1 \%)$ & $14(8.3 \%)$ \\
\hline & 7 & $5(1.2 \%)$ & $4(1.5 \%)$ & $1(0.6 \%)$ \\
\hline
\end{tabular}

Of the 430 embryo transfers analyzed, 262(61\%) elected to have individualized pre-and post-embryo transfer acupuncture performed, while 168(39\%) did not. In order to better assess the effect of acupuncture alone on implantation rates, the potentially confounding variables between the two groups - age, BMI, AMH, PGT, ERA, day of transfer, and embryo morphology were analyzed in the table of demographics and baseline characteristics (Table 1).
A logistic regression model was developed to isolate which of them had an impact on implantation and clinical pregnancy rate. Of the predictor variables listed above, four had statistically significant impacts on transfer success: acupuncture, PGT, BMI, and AMH level as shown in Table 2. These parameters were then adjusted for in a multivariate logistic regression to isolate the effect of acupuncture on implantation. 
Table 2: Multivariate binomial logistic regression model adjusting for age, BMI, AMH, day of transfer, PGT, ERA, and morphology, with OR of implantation and clinical pregnancy rate. Statistically significant findings italicized.

\begin{tabular}{|c|c|c|c|c|c|}
\hline & & \multicolumn{2}{|c|}{ Implantation } & \multicolumn{2}{|c|}{ Clinical Pregnancy } \\
\hline & & OR (95\% CI) & $\begin{array}{c}\text { p value } \\
(<0.05)\end{array}$ & OR $(95 \% \mathrm{CI})$ & p value \\
\hline \multicolumn{6}{|l|}{ Age groups } \\
\hline & $<35$ & Reference & Reference & Reference & Reference \\
\hline & $35-37$ & $0.85(0.48,1.51)$ & 0.587 & $1.00(0.54,1.83)$ & 0.996 \\
\hline & $38-40$ & $1.17(0.62,2.20)$ & 0.627 & $1.17(0.59,2.29)$ & 0.642 \\
\hline & 41 & $0.63(0.27,1.44)$ & 0.269 & $0.99(0.40,2.46)$ & 0.987 \\
\hline & $\geq 42$ & $1.00(0.49,2.05)$ & 0.994 & $1.62(0.71,3.68)$ & 0.246 \\
\hline \multicolumn{6}{|l|}{ BMI Groups } \\
\hline & $<25$ & Reference & Reference & Reference & Reference \\
\hline & $25-30$ & $0.338(0.16,0.73)$ & 0.005 & $0.31(0.15,0.64)$ & 0.002 \\
\hline & $>30$ & $1.29(0.39,4.32)$ & 0.678 & $1.23(0.40,3.78)$ & 0.718 \\
\hline \multicolumn{6}{|l|}{ Acupuncture } \\
\hline & No & Reference & Reference & Reference & Reference \\
\hline & Yes & $1.64(1.04,2.58)$ & 0.032 & $1.64(1.05,2.56)$ & 0.031 \\
\hline \multicolumn{6}{|l|}{ Day of Transfer } \\
\hline & Day 5 & Reference & Reference & Reference & Reference \\
\hline & Day 3 & $0.13(0.01,2.64)$ & 0.206 & $0.23(0.04,3.23)$ & 0.264 \\
\hline & Day 6 & $1.40(0.27,10.29)$ & 0.623 & $1.47(0.19,11.26)$ & 0.317 \\
\hline & Day 7 & $0.85(0.13,5.88)$ & 0.118 & $0.52(0.07,3.66)$ & 0.293 \\
\hline \multicolumn{6}{|l|}{ Embryo Grade } \\
\hline & Good & Reference & Reference & Reference & Reference \\
\hline & Fair & $0.97(0.54,1.75)$ & 0.916 & $0.79(0.45,1.40)$ & 0.43 \\
\hline \multicolumn{6}{|l|}{ PGT } \\
\hline & No & Reference & Reference & Reference & Reference \\
\hline & Yes & $1.85(1.18,2.90)$ & 0.008 & $1.75(1.12,2.74)$ & 0.013 \\
\hline \multicolumn{6}{|l|}{ ERA } \\
\hline & No & Reference & Reference & Reference & Reference \\
\hline & Yes & $0.65(0.38,1.12)$ & 0.121 & $0.61(0.35,1.04)$ & 0.069 \\
\hline \multicolumn{6}{|l|}{$\mathrm{AMH}$} \\
\hline & First quartile & Reference & Reference & Reference & Reference \\
\hline & Second quartile & $1.46(0.69,3.07)$ & 0.32 & $1.68(0.91,3.13)$ & 0.1 \\
\hline & Third quartile & $1.74(0.94,3.25)$ & 0.08 & $1.53(0.81,2.90)$ & 0.191 \\
\hline & Fourth quartile & $1.94(1.01,3.74)$ & 0.047 & $1.21(0.59,2.50)$ & 0.598 \\
\hline
\end{tabular}

\section{Implantation and clinical pregnancy rates}

The percentage of embryo transfers resulting in implantation was positively associated with the use of acupuncture (Figure 1): $76.7 \%$ in the acupuncture group compared to $65.2 \%$ in the control group in the subset of patients who elected to do preimplantation genetic testing; $62.1 \%$ compared to $51.52 \%$ in the subset of patients without PGT. This difference was found to continue throughout clinical pregnancy rates $(71.1 \%$ compared to $56.5 \%$ in the subset of patients who elected to do PGT; $55.3 \%$ compared to $46.5 \%$ in the subset of patients without PGT). 


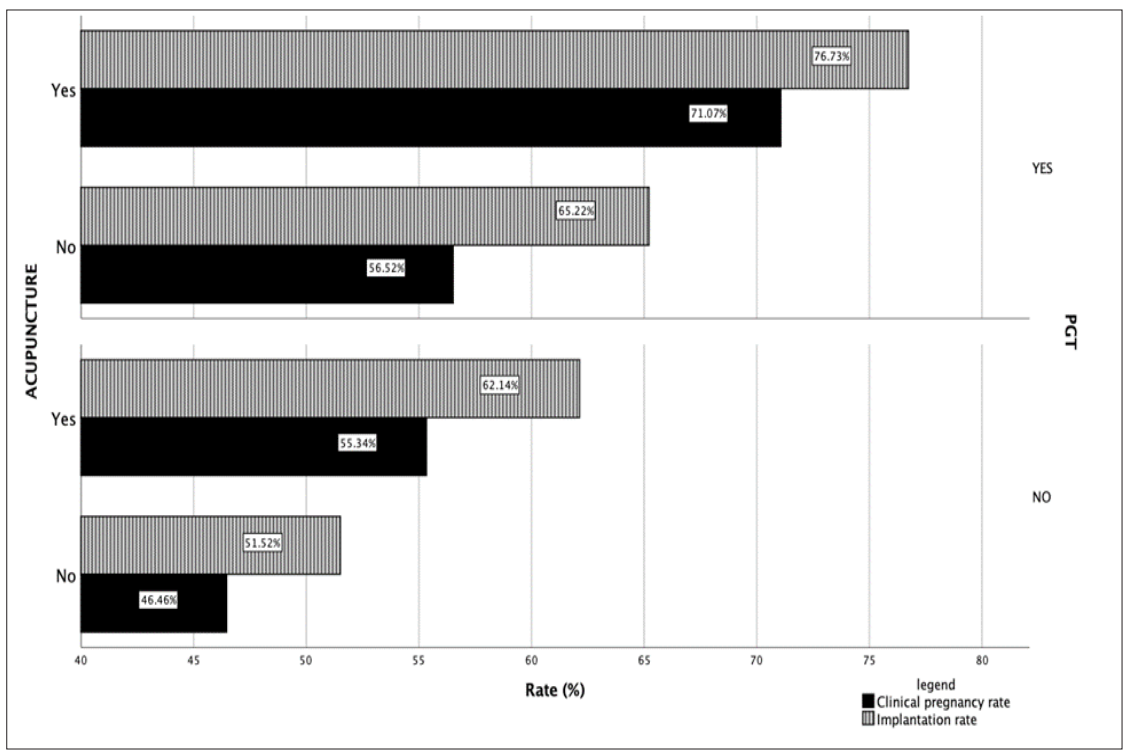

Figure 1: Implantation and clinical pregnancy rates per embryo transfer in each age group.

\section{Isolating the impact of acupuncture}

A multivariate logistic regression model was used to isolate the impact of acupuncture on implantation and clinical pregnancy rates. When compared with the group without acupuncture, significantly increased implantation rates were observed in the acupuncture group (OR 1.64, 95\% CI 1.04-2.58) (Table 2). Clinical pregnancy rates were also noted to be significantly increased (OR 1.64, 95\% CI 1.05-2.56). Of note, the use of PGT was noted to have significantly increased implantation (OR 1.85, 95\% CI 1.18-2.90) and clinical pregnancy rates (OR 1.75, 95\% CI 1.12-2.74). Patients in the uppermost quartile of $\mathrm{AMH}$ level were noted to have significantly increased implantation rates (OR 1.94, 95\% CI 1.01-3.74) but no significant differences in clinical pregnancy rates. Age, embryo grade, day of embryo transfer, and the use of ERA did not cause significant changes in implantation or clinical pregnancy rates.

\section{Discussion}

Our study found that patients undergoing elective, individualized pre-and post-embryo transfer acupuncture on the day of embryo transfer experienced significantly higher implantation and clinical pregnancy rates than those who did not. There are several other studies examining the effects of acupuncture on embryo transfer, finding that acupuncture before and after embryo transfer had effects such as increasing implantation success or decreasing maternal anxiety levels [5,6]. Furthermore, a systematic review and meta-analysis found that acupuncture may be effective as compared to a non-treatment control, while the effect was less strong compared to a sham control [7]. Our study controlled for potential confounding factors such as age, BMI, AMH, PGT, ERA, embryo morphology, and day of transfer, in order to better isolate the impact of acupuncture alone on implantation success rate. It is important to separate the dataset between pre-implantation genetic testing for aneuploidy (PGT-A) and non-PGT-A tested embryos when evaluating implantation and clinical pregnancy rates. This allows the strong confounding effect of aneuploid embryos on implantation to be eliminated. The multivariate logistic regression analysis performed allows us to determine that the effect of acupuncture is independent not only of ploidy, as we did separate the patients into PGT-A tested and not tested groups, but also of other variables known to be detrimental to implantation such as age, BMI, AMH, embryo morphology, and day of transfer [17]. Interestingly, ERA was not shown to cause improvement in implantation rates. ERA attempts to assess the degree of endometrial receptivity to implantation, evaluating for an endometrial window of implantation regardless of histological appearance [18]. This assay was therefore expected to positively impact implantation rates. The lack of benefit in this study is because patients who choose to perform a mock cycle for ERA are usually ones who have had multiple failed transfers for other unknown reasons. Uterine factors, such as fibroids, intrauterine adhesions, and polyps, are routinely evaluated for and treated prior to implantation at the Vios Fertility Institute. Therefore, uterine factors such as these are not likely to have an impact upon implantation and clinical pregnancy rates in patients from this clinic.

The cause of improvement in implantation and clinical pregnancy rates due to acupuncture is largely unclear at this time, however, theories include acupuncture's impact on the hypothalamic-pituitary-gonadal axis and uterine blood flow. It is believed that acupuncture increases release of beta-endorphin and serotonin, [8] which then increase pulsatile GnRH secretion. GnRH then acts on the pituitary gland to increase release of LH and FSH, which consequently impact steroid release and reproductive function [12]. These factors are hypothesized to positively influence ovarian follicular growth, ovulation, and 
fertility [10-13]. Acupuncture has additionally been hypothesized to decrease sympathetic activity [13]. This may impact uterine blood flow and therefore endometrial growth and thickness. Increased endometrial thickness, up to a certain point, has been associated with improved implantation and live pregnancy rates [19]. Furthermore, acupuncture may have a positive impact on implantation and live pregnancy rates via psychological factors such as stress reduction, placebo effect, or yet undescribed mechanisms [4]. In order to further expand our understanding of the effects and use of acupuncture on embryo transfer success, further studies will evaluate the impact of acupuncture on live birth rates, as well as cost-effectiveness of these treatments.

\section{References}

1. Jishun J, Mittelman M (2014) Acupuncture: Past, present, and future. SAGE Publications Sage, Los Angeles, CA.

2. Chernyak GV, Sessler DI, Warltier DC (2005) Perioperative acupuncture and related techniques. Anesthesiology 102(5): 1031-1049.

3. Organization WH (2002) Acupuncture: Review and analysis of reports on controlled clinical trials: World Health Organization, WHO, Switzerland.

4. Westergaard LG, Mao Q, Krogslund M, Sandrini S, Lenz S, et al. (2006) Acupuncture on the day of embryo transfer significantly improves the reproductive outcome in infertile women: A prospective, randomized trial. Fertil Steril 85(5): 1341-1346.

5. Guven PG, Cayir Y, Borekci B (2020) Effectiveness of acupuncture on pregnancy success rates for women undergoing in vitro fertilization: A randomized controlled trial. Taiwan J Obstet Gynecol 59(2): 282-286.

6. Dehghani AS, Homayouni K, Kanannejad Z, Kanannejad Z (2020) The effect of acupuncture on the day of embryo transfer on the in vitro fertilization outcomes: An RCT. Int J Reprod Biomed 18(3): 209-214.

7. Smith CA, Armour M, Shewamene Z, Tan HY, Norman RJ, et al. (2019) Acupuncture performed around the time of embryo transfer: A systematic review and meta-analysis. Reprod Biomed Online 38(3): 364-379.
8. Petti F, Bangrazi A, Liguori A, Reale G, Ippoliti F (1998) Effects of acupuncture on immune response related to opioid-like peptides. J Tradit Chin Med 18(1): 55-63.

9. Chang R, Chung PH, Rosenwaks Z (2002) Role of acupuncture in the treatment of female infertility. Fertil Steril 78(6): 1149-1153.

10. Ferin M, Wiele RV (1984) Endogenous opioid peptides and the control of the menstrual cycle. Eur J Obstet Gynecol Reprod Biol 18(5-6): 365-373.

11. Petraglia F, Di Meo G, Storchi R, Segre A, Facchinetti F, et al. ( 1987) Proopiomelanocortin-related peptides and methionine enkephalin in human follicular fluid: changes during the menstrual cycle. Am J Obstet Gynecol 157(1): 142-146.

12. Marshall JC, Kelch RP (1986) Gonadotropin-releasing hormone: Role of pulsatile secretion in the regulation of reproduction. $\mathrm{N}$ Engl J Med 315(23): 1459-1468.

13. Victorin SE, Waldenstrom U, Andersson SA, Wikland M (1996) Reduction of blood flow impedance in the uterine arteries of infertile women with electro-acupuncture. Hum Reprod 11(6): 1314-1317.

14. Roque M, Valle M, Guimarães F, Sampaio M, Geber S (2015) Freezeall policy: Fresh vs. frozen-thawed embryo transfer. Fertil Steril 103(5):1190-1193.

15. Lensen SF, Wilkinson J, Leijdekkers JA, La Marca A, Mol BWJ, et al. (2018) Individualized gonadotropin dose selection using markers of ovarian reserve for women undergoing in vitro fertilisation plus intracytoplasmic sperm injection (IVF/ICSI). Cochrane Database Syst Rev 2(2).

16. (2019) Technology SfAR. SART National Summary Report 2017.

17. Reig A, Franasiak J, Scott RT, Seli E (2020) The impact of age beyond ploidy: Outcome data from 8175 euploid single embryo transfers. Journal of Assisted Reproduction and Genetics 37: 595-602.

18. Gimeno DP, Alonso RM, Blesa D, Bosch N, Conejero MJA, et al. (2013) The accuracy and reproducibility of the endometrial receptivity array is superior to histology as a diagnostic method for endometrial receptivity. Fertil Steril 99(2): 508-517.

19. Shaodi Z, Qiuyuan L, Yisha Y, Cuilian Z (2020) The effect of endometrial thickness on pregnancy outcomes of frozen-thawed embryo transfer cycles which underwent hormone replacement therapy. PloS one 15(9): e0239120. 